\title{
Support-shape dependent catalytic activity in Pt/alumina systems using ultra-small (USANS) and small angle neutron scattering (SANS)
}

\author{
Sang Hoon Kim ${ }^{a}$, Sugyeong Han ${ }^{b}$, Heonphil Ha ${ }^{a}$, Ji Young Byun ${ }^{a}$, Man-Ho Kim ${ }^{\text {b,* }}$ \\ a Center for Materials Architecturing, Korea Institute of Science and Technology, Seoul 136-791, Republic of Korea \\ ${ }^{\mathrm{b}}$ Advanced Analysis Center, Korea Institute of Science and Technology, Seoul 136-791, Republic of Korea
}

\section{A R T I C L E I N F O}

\section{Article history:}

Received 27 February 2015

Received in revised form 19 May 2015

Accepted 28 May 2015

Available online $\mathrm{xxx}$

\section{Keywords:}

Shape

Catalytic activity

Support materials

Ultra small angle neutron scattering

Small angle neutron scattering

\begin{abstract}
A B S T R A C T
The correlation between the morphology of catalyst supports and their effect on catalyst dispersion was investigated. The structure of two types of $\gamma$-alumina $\left(\gamma-\mathrm{Al}_{2} \mathrm{O}_{3}\right)$ crystallite particles, rod-like and plateletlike alumina, were measured in 4 orders of length scales, from nanometer to micrometer, using ultra small angle neutron scattering (USANS) and SANS and quantitatively characterized with Hammouda's Generalized Guinier-Porod model. Pt nanoparticles tended to deposit in finer particles and had narrower particle size distribution on the rod-like alumina supports compared to the plate-like alumina supports due to geometrically restricted deposition areas and higher surface curvatures of the rod-like supports. The high diffusion barrier for Pt particles on the highly curved surfaces of the rod-like supports was attributed to be the reason why Pt particles were prevented from diffusing and clustering. While aggregates of the rod-like alumina supports were randomly dispersed without any specific orientation, resulting in high surface area, aggregates of the plate-like alumina supports consisted of a stack of 2-3 layers due to the high van der Waals forces between planar layers, resulting in low surface area. Pt/rod-like alumina supports showed more than $100 \%$ higher catalytic activities than Pt/platelet-like alumina supports in model three-way-catalyst (TWC) reactions of $\mathrm{CO}, \mathrm{NO}$, and $\mathrm{C}_{3} \mathrm{H}_{6}$ conversions at $200-250^{\circ} \mathrm{C}$. Control of shape and aggregation of catalytic support materials in nano-micrometer scale can be an important parameter to improve catalytic performance.
\end{abstract}

(c) 2015 Elsevier B.V. All rights reserved.

\section{Introduction}

There are many factors that determine the catalytic performance of oxide supported metal catalysts. Many studies have been devoted to maximize the metal-support interaction and exposed surface area of deposited catalyst materials [1-3]. There is a strong need to maximize the catalytic performance for a given amount of catalyst materials as they are expensive precious metals (Pt, Pd, $\mathrm{Ph}$, etc). The effect of the Pt size and shape on lean reduction of NO with $\mathrm{C}_{3} \mathrm{H}_{6}$ was studied, showing that catalytic activity was affected by the change in morphology of the Pt nanoparticles during the reaction [4]. As for catalytic support materials, there have been many studies for increasing the catalytic reactivity of the support itself through nanometer scale engineering, doping or mixing foreign materials to the support materials [5]. Apart from such modifications of the chemical nature of the support materials, it is also possible that the catalytic performance can vary significantly

\footnotetext{
* Corresponding author. Tel.: +82 29585395.

E-mail address: man-hokim@kist.re.kr (M.-H. Kim).
}

depending on the structural characteristics of the support material such as its porosity, pore size distribution, specific surfaces, etc. However, less attention is paid to the structural aspects of support materials in terms of improving the catalytic performance than their chemical nature. Although there are a few papers in the literature regarding the influence of preparation processes or parameters of $\gamma$-alumina on its final shape [6,7], those studies focused on the lattice structures in atomic level only, but not the shape or aggregates in nano-meso-micron scale. Also, we could not find any study directly relating shapes of $\gamma$-alumina as catalytic support to its influence on the catalytic activity. In order to correlate the structure and the catalyst activity of the support material, qualitative and quantitative information on the support materials is necessary. Ultra small angle scattering (USANS) and small angle neutron scattering (SANS) techniques are powerful tools to measure particle and pore size, their shapes, correlation length between two scatters, and surface structure.

Porous $\gamma$-alumina powder is one of the most widely used catalyst support materials in automobile catalysts [8]. However, the shape and structure in different length scales and their correlation with catalytic activity has not been studied. In this study, we 
investigated the structural aspect of Pt/alumina systems in different length scales, from nanometers to micrometers, using the USANS and SANS measurements and compared the catalytic reactivity of the systems with different shaped $\gamma$-alumina powders.

\section{Experimental}

\subsection{Materials and catalytic reactions}

Two different types of $\gamma$-alumina powder, having negligible difference in the chemical properties of the surface (see Supplementary information for X-ray photoelectron spectroscopy (XPS) result), were used as catalyst support materials. The first one was research grade from Alfar aesar (AA) with a high surface area $\left(255 \mathrm{~m}^{2} / \mathrm{g}\right.$ from BET); the second one was for industrial usage as a commercial catalyst support from AEROXIDE (AO) Alu C with a typical surface area $\left(130 \mathrm{~m}^{2} / \mathrm{g}\right.$ from BET).

$\mathrm{Pt} /$ alumina complex samples were prepared by depositing $\mathrm{Pt}$ nanoparticles by arc plasma deposition (APD) at room temperature under $10^{-5}$ Torr vacuum [9]. In the APD system (ULVAC, ARL-300), a cylindrical cathode rod made of target materials $(\mathrm{Pt})$ and a trigger electrode is placed at the center and a cylindrical anode is coaxially mounted, wrapping the cathode. A defined amount of charge is stored in a discharge condenser that is connected to the cathode. When a trigger pulse induces an arc discharge between the surface of a target rod (cathode) and an anode, an accumulated charge flows from a cathode spot to an anode via an arc for a short time, typically less than $1 \mathrm{~ms}$. During the process, the cathode spot temperature rises more than tens of thousands of degrees Celsius, vaporizing the cathode material and generating ionized plasma pulses with high kinetic energies $(10 \mathrm{~km} / \mathrm{s})$. During the process, nanoparticles with a typical size of $1-5 \mathrm{~nm}$ are generated. Right after the discharge, the condenser is quickly charged up again for the next plasma pulse generation. In the process, controllable parameters are the discharge condenser voltage $V$, the discharge condenser capacity $C$, and the number of plasma pulse shots $n$.

Catalytic performance was measured in a flow reactor. The catalyst samples underwent model reactions of the three way catalyst (TWC) reactions ( $\mathrm{CO}, \mathrm{NO}, \mathrm{C}_{3} \mathrm{H}_{6}$ conversions) in a flow reactor [10]. $\mathrm{O}_{2}, \mathrm{CO}, \mathrm{NO}$, and $\mathrm{C}_{3} \mathrm{H}_{6}$ gases were used as reactant gases with $\mathrm{N}_{2}$ gas as carrier gas. The total gas flow was $300 \mathrm{~cm}^{3} / \mathrm{min}$ and the sample volume was $1 \mathrm{~cm}^{3}$ (space velocity of $18,000 \mathrm{~h}^{-1}$ ). $\mathrm{O}_{2}$ gas was kept at $1 \mathrm{vol} \%, \mathrm{NO}_{x}$ (NO with small $\mathrm{NO}_{2}$ trace) at $500 \mathrm{ppm}, \mathrm{CO}$ at $1000 \mathrm{ppm}$, and $\mathrm{C}_{3} \mathrm{H}_{6}$ at $500 \mathrm{ppm}$, which is a lean condition [11,12]. The sample catalysts were preheated at $300^{\circ} \mathrm{C}$ for $1 \mathrm{~h}$ before being exposed to the reactant gases. The conversion rate $r$ was calculated as $r=\left(C_{\text {in }}-C_{\text {out }}\right) / C_{\text {in }} \times 100$, where $C_{X}$ is the concentration of $x$ species.

\subsection{USANS and SANS measurements}

The $\gamma$-alumina powder samples were loaded into quartz cells with a path length of $1 \mathrm{~mm}$ and a diameter of $12.5 \mathrm{~mm}$. The circular surface of the cell was placed perpendicular to the incoming neutron beam. USANS data were measured using the recently commissioned KIST-USANS instrument for cold neutrons on the CG4B at the HANARO Cold Neutron Facility. The transmissions of samples were around $80 \%$, which confirms no multiple scattering. The instrument is comprised of a pair of multiple-bounce channel-cut perfect $\mathrm{Si}\left(\begin{array}{lll}1 & 1 & 1\end{array}\right)$ crystals. One acts as a monochromator and the other acts as an analyzer. A wavelength of $\lambda=4 \AA$ with a wavelength resolution of $\Delta \lambda / \lambda \sim 11 \%$ was used. The USANS scattering intensities were measured in the range of scattering vectors, $2 \times 10^{-5} \AA^{-1}<Q(=(4 \pi \sin \theta) / \lambda)<\sim 2 \times 10^{-2} \AA^{-1}$, where $2 \theta$ is the scattering angle. This was done by rotating the analyzer in an angle-by-angle manner to match the Bragg angle of the analyzer to the scattering angle from the sample. The USANS scattering intensities of the samples were converted to the smeared total cross-section (unit: $\mathrm{cm}^{-1} \mathrm{str}^{-1}$ ) by subtracting the empty scattering and normalizing with respect to the sample transmission and thickness. The smeared total cross-sections $\mathrm{d} \Sigma / \mathrm{d} \Omega(Q)_{\mathrm{sm}}$ of the USANS data were desmeared by raising the scattering power by one (i.e., $\left.\mathrm{d} \Sigma / \mathrm{d} \Omega(Q)_{\mathrm{sm}} \times \Delta q_{V} / Q\right)$ to match the SANS cross-sections, $\mathrm{d} \Sigma / \mathrm{d} \Omega(Q)$, where $\Delta q_{V}$ is the vertical divergence of the detector.

Small Angle Neutron Scattering (SANS) data were measured using the $18 \mathrm{~m}$ SANS instrument of the cold neutron guide CG4B at the HANARO Cold Neutron Facility. Two sample-to-detector distances of $3 \mathrm{~m}$ and $9 \mathrm{~m}$ were used with a cold neutron wavelength of $\lambda=4.45 \AA$, and $\lambda=8.28 \AA$. The scattering intensities were measured in the range of scattering vectors $Q \sim 0.00464 \AA^{-1}$ to $Q \sim 0.2 \AA^{-1}$ with a beam diameter of $10 \mathrm{~mm}$. The measured intensities, $I(Q)$, were converted to the absolute scale (i.e., total cross-sections, $\mathrm{d} \Sigma / \mathrm{d} \Omega(Q))$ by correcting them with a standard silica sample with the known $R_{g}$ and $I(0)$.

Both USANS and SANS profiles in absolute scale were well combined to cover $Q$ from $Q=2 \times 10^{-5}$ to $\sim 2 \times 10^{-1} \AA^{-1}$, corresponding to sizes from approximately a micrometer to a nanometer.

\subsection{Transmission electron microscopy}

Dispersed Pt nanoparticles on $\gamma$-alumina powder were observed by Transmission Electron Microscopy (TEM). TEM investigations were performed using a JEM $2100 \mathrm{~F}$ (JEOL Co.) electron microscope with $2 \AA$ point-to-point resolution and an acceleration voltage of $200 \mathrm{kV}$. To measure the size distribution of deposited Pt nanoparticles, about $100 \mathrm{Pt}$ nanoparticles in each TEM image were randomly selected and their diameters were measured by ImageJ (NIH). The average particle size and its standard deviation were calculated from the measured diameter values. The error range for the diameter measurement was about $10 \%$.

\subsection{Theoretical background of quantitative characterization}

The size of particles, such as the radius of gyration $R_{g}$, is typically characterized with a Guinier approximation in small angle scattering when the scattering profiles show a knee, where the scattering profile bends downward. Depending on the shape of the particles, Guinier's approximate form factor, $P(Q)$, is expressed as follows (see, Chapter 2 by G. Porod) [13]:

$$
\begin{array}{r}
P(Q)_{\mathrm{sph}}=(V \cdot \Delta \mathrm{SLD})^{2} \exp \left(-\frac{Q^{2} R_{g}^{2}}{3}\right) \\
\text { for } \quad Q R_{g} \leq 1 \quad \text { (sphere) }
\end{array}
$$

$$
\begin{gathered}
P(Q)_{\mathrm{plt}}=(t \cdot \Delta \mathrm{SLD})^{2} \frac{2 \pi \cdot A_{\mathrm{plt}}}{Q^{2}} \exp \left(-Q^{2} R_{g t}^{2}\right) \\
\text { for } Q R_{g t} \leq 1 \quad \text { (platelet) } \\
P(Q)_{\text {rod }}=\left(A_{\text {rod }} \cdot \Delta \mathrm{SLD}\right)^{2} \frac{\pi \cdot L}{Q} \exp \left(-\frac{Q^{2} R_{g c}^{2}}{2}\right) \\
\text { for } Q R_{g c} \leq 1 \quad(\operatorname{rod})
\end{gathered}
$$

where $R_{g}, R_{g t}$, and $R_{g c}$ are the radius of gyration for spheres, the thickness radius of gyration in platelets, and cross-sectional radius of gyration in rods, respectively. $A_{\text {plt }}$ is the area of the platelet planes and $L$ is the length of the rods. The other symbols, $V, t, A_{\text {rod }}$, and $\Delta S L D$ represent the volume of the spheres, the thickness of the platelets, the cross-section of the rods, and the difference of the scattering 
length densities between two domains, respectively. Eqs. (1)-(3) can be simplified as follows [14]:

$$
\begin{aligned}
& P(Q)_{G_{i}}= \frac{G_{i j}}{Q^{s_{i j}}} \exp \left(-\frac{Q^{2} R_{g_{i j}}^{2}}{3-s_{i j}}\right) \\
& \text { for }\left\{\begin{array}{l}
Q R_{g_{i j}} \leq 1, \quad Q \leq Q_{i 2}, \quad Q_{i 2} \leq Q \leq Q_{i 1} \\
i=0 \text { (spheres), } \quad i=1 \text { (rods), } \quad i=2 \text { (platelets) } \\
j=1(1 \text { st Guinier) }, \quad j=2 \text { (2nd Guiner) }
\end{array}\right.
\end{aligned}
$$

where $s_{i j}$ is a dimensionality parameter to determine the shape of a scattering entity; $s_{01}=0$ for spheres, $s_{11}=1$ for rods, and $s_{21}=2$ for lamellae while $s_{02}=s_{12}=s_{22}=0$ for spheres, rods, and lamellae with an equal lateral dimension. $R_{g_{i 1}}$ is a characteristic dimensional radius of gyration in Eqs. (1)-(3) like $R_{g}, R_{g t}, R_{g c}$. $G_{i j}$ is a Guinier scale factor depending on the shape of scatters:

$G_{i j}= \begin{cases}(V \cdot \Delta \mathrm{SLD})^{2} & \text { for } \text { spheres } \\ (t \cdot \Delta \mathrm{SLD})^{2} s_{21} \pi A_{\mathrm{plt}} & \text { for } \text { platelets } \\ (A \cdot \Delta \mathrm{SLD})^{2} s_{11} \pi L & \text { for } \text { rods }\end{cases}$

Short dimensions, the cross-section diameter $D$ of a rod, the thickness $t$ of a platelet as well as the diameter $D_{\text {sph }}$ of a sphere can be determined from the corresponding radius of gyration (see Chapter 4 by 0 . Glatter in the reference of $[13,15]$ :

$$
\begin{aligned}
& D_{\mathrm{sph}}=2 \sqrt{\frac{5}{3}} R_{g 01} \\
& D=2 \sqrt{2} R_{g 11} \\
& t=2 \sqrt{3} R_{g 21}
\end{aligned}
$$

Long dimensions, the length $L$ of rods and the width $W$ of platelets can be obtained from the length radius of gyration, $R_{g 21}$, and width radius of gyration, $R_{g 22}$ :

$$
\begin{array}{ll}
L=2 \sqrt{3 \cdot\left(R_{g 12}^{2}-R_{g 11}^{2}\right)} \approx 2 \sqrt{3 R_{g 11}^{2}} \text { for } \quad R_{g 12} \gg R_{g 11} \\
W=2 \sqrt{3 \cdot\left(R_{g 22}^{2}-R_{g 21}^{2}\right)} \approx 2 \sqrt{3 R_{g 22}^{2}} \text { for } \quad R_{g 22} \gg R_{g 21}
\end{array}
$$

Finding the right Guinier region may be difficult when the Guinier tail is interfered by a power law scattering.

While the Guinier scattering region allows for the measurement of the size of scatters and the identification of the shape, the power law scattering $I(Q)_{P}$ that decreases with a power index, $p$, with $Q$, contains information about the surface of scatters as follows:

$I(Q)_{P}=K Q^{-p}\left\{\begin{array}{l}p=4 \text { (smooth \& sharp interface) } \\ 1 \leq p<3 \text { (mass (i.e., volume) fractal) } \\ p=3 \text { (uniform globular object or limit of rougher } \\ 3 \leq p<4 \text { (surface fractal) } \\ p>4 \text { (diffused interfacial boundary) }\end{array}\right.$

The Porod law $(p=4)$ representing a smooth surface is a special case of power law scattering from a surface. Guinier and power law scattering require individual curve fitting to characterize the scattering curves. Therefore, it is imperative to unify the two scattering laws to a single formula so that it characterizes both Guinier and Power law scatterings simultaneously. Hammouda successfully showed that two independent scatterings, the first Guinier scattering $P(Q)_{G 1}$ at $Q \leq Q_{\mathrm{GP}}$ followed by power law scattering $P(Q)_{P}$ at $Q \geq Q_{\mathrm{GP}}$, can be unified to a single Guinier-Porod (GP) formula, when a scattering curve changes continuously at a scattering vector $Q_{G P}$ with the following conditions [14]:

$$
\begin{aligned}
& P\left(Q_{G P}\right)_{G 1}=I\left(Q_{G P}\right)_{P} \\
& \left.\frac{\mathrm{d} P(Q)_{G 1}}{\mathrm{~d} Q}\right|_{Q_{G 1 P}}=\left.\frac{\mathrm{d} I(Q)_{P}}{\mathrm{~d} Q}\right|_{Q_{G 1 P}} \text { for } \quad\left(Q \geq Q_{\mathrm{GP}}\right)
\end{aligned}
$$

The diameter $D$ and thickness $t$ (i.e., short dimensions) of the rod-like and platelet-like shapes are obtained from the first Guinier region. When the second Guinier scattering $P(Q)_{G 2}$ at $Q \leq Q_{G G}$ originated from the overall dimensions (i.e., mainly from large dimensions because of negligible small dimension) are followed by the first Guinier scattering $P(Q)_{G 1}$ at $Q \geq Q_{G G}$, two continuing Guinier scattering regions can be expressed as follows:

$P\left(Q_{G G}\right)_{G 2}=P\left(Q_{G G}\right)_{G 1}$

and $\left.\quad \frac{\mathrm{d} P(Q)_{\mathrm{G}_{2}}}{\mathrm{~d} Q}\right|_{Q_{2}}=\left.\frac{\mathrm{d} P(Q)_{\mathrm{G}_{1}}}{\mathrm{~d} Q}\right|_{\mathrm{Q} 2} \quad$ for $\quad Q \leq Q_{\mathrm{GG}}$

The Porod constant, $K_{P}$, from Eqs. (4), (9), and (10) is:

$K_{P}=G_{i 1} \exp \left(\frac{-Q_{1}^{2} R_{g i 1}^{2}}{3-s_{i 1}}\right) \cdot Q_{1}^{\left(p-s_{i 1}\right)}\left(p>s_{i 1}, 3>s_{i 1}\right)$

and the transition points of $Q_{\mathrm{GP}}$ from Guinier to power scattering and $Q_{\mathrm{GG}}$ Guinier and Guinier regions follow [14]:

$$
\begin{gathered}
Q_{\mathrm{GG}}=\sqrt{\frac{\left(s_{i 1}-s_{i 2}\right)}{\left(2 R_{g i 2}^{2} /\left(3-s_{i 2}\right)\right)-\left(2 R_{g i 1}^{2} /\left(3-s_{i 1}\right)\right)}} \\
\text { for } \quad 3>s_{i 1}>s_{i 2}, \quad R_{g i 2}>R_{g i 1}
\end{gathered}
$$

$Q_{\mathrm{GP}}=\frac{1}{R_{g}} \sqrt{\frac{\left(p-s_{i 1}\right)\left(3-s_{i 1}\right)}{2}}$

$G_{\mathrm{GG}}=G_{\mathrm{GP}} \exp \left[-Q_{\mathrm{GG}}^{2}\left(\frac{R_{g i 1}^{2}}{3-s_{i 1}}-\frac{R_{g i 2}^{2}}{3-s_{i 2}}\right)\right] Q_{G}^{\left(s_{i 2}-s_{i 1}\right)}$

where the scaling factor, $G_{\mathrm{GP}}$, in Eq. (17) is an input parameter for fitting. Table 1 summarizes the relationships among the parameters of the GP and GG fittings.

\section{Results and discussion}

3.1. Characterization of alumina aggregates using USANS and SANS measurements

Microscopic structures of the two types of $\gamma$-alumina, AA and AO, were characterized with USANS and SANS measurements.
surface)
for $Q \geq Q_{G P}$

Independent measurements of the USANS and SANS superimpose in the double logarithm plot of the total cross-section against $Q$ (Fig. 1 for AA and Fig. 2 for AO). The USANS-SANS scattering profiles cover more than 11 orders in magnitude of total scattering cross-sections ( $y$-axis, $(\mathrm{d} \Sigma / \mathrm{d} \Omega)(Q))$ and 4 orders in $Q$ scale, which range from nanometers to micrometers in size. The different features in the slopes and knees of the scattering curves of the two pure $\mathrm{Al}_{2} \mathrm{O}_{3}$ powders were observed in the wide $Q$ range, indicating differences in the structure and shape of the scattering entities. Characterizations of the scattering curves are shown in Figs. 1 and 2. 
Table 1

List of parameters related to the generalized Guinier-Power (GP) and Guinier-Guinier(GG) fit.

\begin{tabular}{|c|c|c|c|c|c|c|c|c|c|c|}
\hline & \multicolumn{2}{|c|}{$s_{i j}$} & \multirow[t]{2}{*}{$p$} & \multirow[t]{2}{*}{$Q_{\mathrm{GP}}\left(\AA^{-1}\right)$} & \multirow[t]{2}{*}{$Q_{G G}\left(\AA^{-1}\right)$} & \multirow[t]{2}{*}{$R_{\text {gi1 }}(\AA)$} & \multirow[t]{2}{*}{$R_{\mathrm{gi} 2}(\AA)$} & \multirow[t]{2}{*}{ Dimensions $(\AA)$} & & \multirow[t]{2}{*}{$S / V\left(\mathrm{~m}^{2} / \mathrm{g}\right)$} \\
\hline & $S_{\mathrm{i} 1}$ & $S_{\mathrm{i} 2}$ & & & & & & & & \\
\hline Sphere $(i=0)$ & 0 & 0 & - & - & - & $\sqrt{(3 / 5) R}$ & - & $D_{\mathrm{sph}}=2 \sqrt{(5 / 3) R_{g 01}}$ & & - \\
\hline \multirow{2}{*}{$\begin{array}{l}\text { Rod } \\
(i=1)\end{array}$} & \multirow[t]{2}{*}{1} & \multirow[t]{2}{*}{0} & \multirow[t]{2}{*}{-} & \multirow{2}{*}{$\sqrt{3} / R_{g 11}$} & \multirow{2}{*}{$\sqrt{\frac{3}{2}} \frac{1}{R_{g 12}}$} & \multirow{2}{*}{$r / \sqrt{2}$} & \multirow[t]{2}{*}{$(L+r) / \sqrt{12}$} & $D(\AA)$ & $L(\AA)(L \gg r)$ & - \\
\hline & & & & & & & & $2 \sqrt{2} R_{g 11}$ & $\sqrt{12} R_{g 12}$ & $\frac{2 L+D}{r L}$ \\
\hline \multirow[t]{2}{*}{ Platelet $(i=2)$} & \multirow[t]{2}{*}{2} & \multirow[t]{2}{*}{0} & \multirow[t]{2}{*}{-} & \multirow[t]{2}{*}{$1 / R_{g 21}$} & \multirow[t]{2}{*}{$\sqrt{3} / R_{g 22}$} & \multirow[t]{2}{*}{$t / \sqrt{12}$} & \multirow[t]{2}{*}{$(W+t) / \sqrt{12}$} & $t(\AA)$ & $W(\AA)(W \gg r)$ & \\
\hline & & & & & & & & $\sqrt{12} R_{\mathrm{g} 21}$ & $\sqrt{12} R_{\mathrm{g} 22}$ & $(8 W+t) / W^{2} t$ \\
\hline
\end{tabular}

Figs. 1 and 2 show at least three distinctive slopes along the entire $Q$ region. In Fig. 1 , the slope of -1 observed around $Q \sim 10^{-2}-7 \times 10^{-2} \AA^{-1}$ is characteristic of one dimensional shapes (Eqs. ( 3 ) and (4)). In Fig. 2, the slope of -2 covering almost 2 orders in the $Q$ scale (i.e., $Q \sim 6 \times 10^{-4} \AA^{-1}$ and $2 \times 10^{-2} \AA^{-1}$ ) is characteristic of two dimensional shapes (Eqs. (2) and (4)). Despite the same chemical composition and crystal structure (i.e., $\gamma$ phase alumina crystallites) of the two pure alumina powders, they are different in shape. Pure-AA alumina is rod-like and pure-AO alumina is plateletlike in shape. The scatterings at the Guinier-Porod transition, $Q_{G P}$, are from primary scatterers related to small dimensions, diameter in rods and thickness in platelets.

The large dimensions, like the overall size (including the length and width of rods and platelets) must appear in the second Guinier region, which is at a lower scattering angle than the first Guinier region representing the diameter and the thickness. However, the second Guinier regions from the large dimensions are hidden underneath strong low angle upturns, below $Q \sim 2 \times 10^{-4} \AA^{-1}$ for AA and below $Q \sim 6 \times 10^{-3} \AA^{-2}$ for AO, originating from the aggregates of crystallite particles (Figs. 1 and 2). Assuming that the GG transition starts around the upturn, where the sum of power law scatterings and the 2nd Guinier region is equal to the observed upturn scatterings, we fit the data by adding the GG code to GP model program in the NIST SANS data analysis package [16]. The

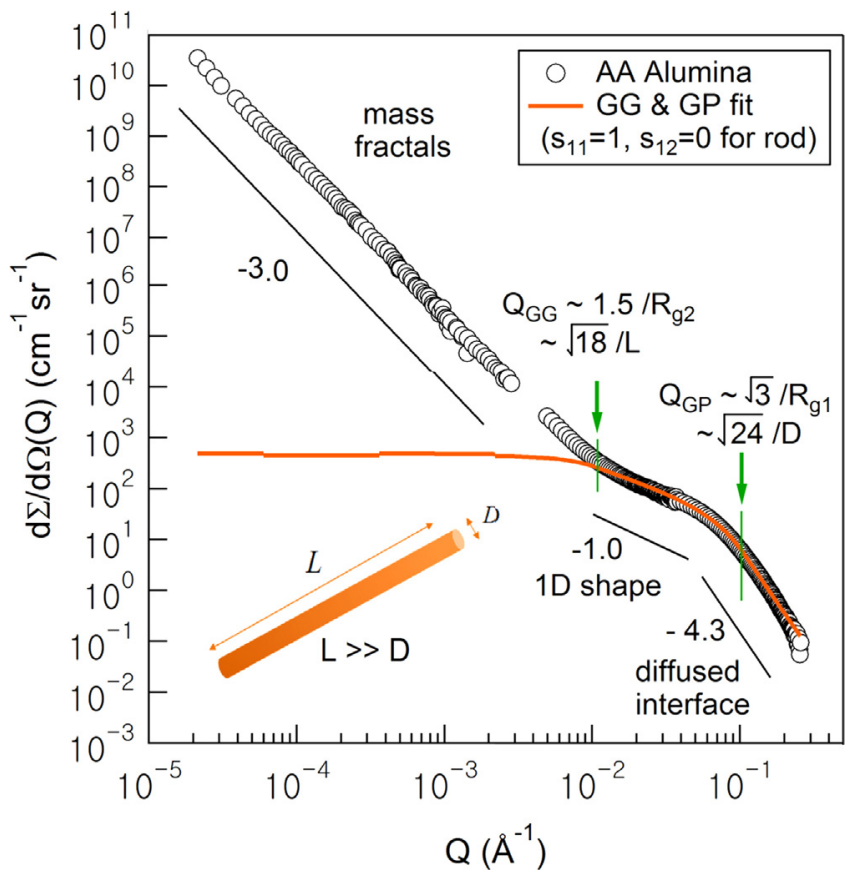

Fig. 1. USANS and SANS profiles (open circle) of pure AA alumina and generalized Guinier-Power and Guinier-Guinier fit (line) with $s_{11}=1, s_{12}=0$. Inset is a rod shape. fittings (the orange line) for short and overall dimensions of rods and platelets are shown Figs. 1 and 2. The GP transition points were determined as $Q_{\mathrm{GP}}=0.105 \AA^{-1}$ for the cross sectional diameter of $A A$ rods and $Q_{G P}=1.038 \times 10^{-2} \AA^{-1}$ for the thickness of $A O$ platelets. The corresponding radii of gyration and the short dimensions are $R_{g c}=16.8 \AA$ and $D=47.5 \AA$ for AA alumina and $R_{g t}=31.8 \AA$ and $t=110 \AA$ for $A O$ alumina. The GG transition points are estimated as $Q_{G G}=0.01021 \AA^{-1}$ and $Q_{G G}=6.9282 \times 10^{-4} \AA^{-1}$ for the rods and platelets, respectively. The corresponding large dimensions are $L \sim 412 \AA$ for $\mathrm{AA}$ rods and $W \sim 8660 \AA$ for $\mathrm{AO}$ platelets. Table 2 lists the structural parameters of $A A$ and $A O$ alumina obtained with the relationships among the parameters summarized in Table 1.

In addition to the GP and GG scatterings, strong low angle upturns appear below $Q_{G 1 G 2}$. The AO alumina show two low angle scatterings with $p=1.8$ below $Q=\sim 5 \times 10^{-5} \AA^{-1}$ followed by $p=2.8$ in the $Q=\sim 6 \times 10^{-5}-2 \times 10^{-4} \AA^{-1}$. On the other hand, the AA alumina show a single low angle scattering with a single power index of $p=3$ covering the broad $Q$ range of $2 \times 10^{-5}-5 \times 10^{-3} \AA^{-1}$. The excess intensities at low $Q$ reflect aggregation of individual alumina particles (i.e., aggregation of individual alumina for rod-like alumina and of stacked alumina for plate-like alumina). A power index $p$ in $1<p<d$ indicates a mass (or volume) fractal structure, where $d$ is a Euclidean dimension. For example, a power index in

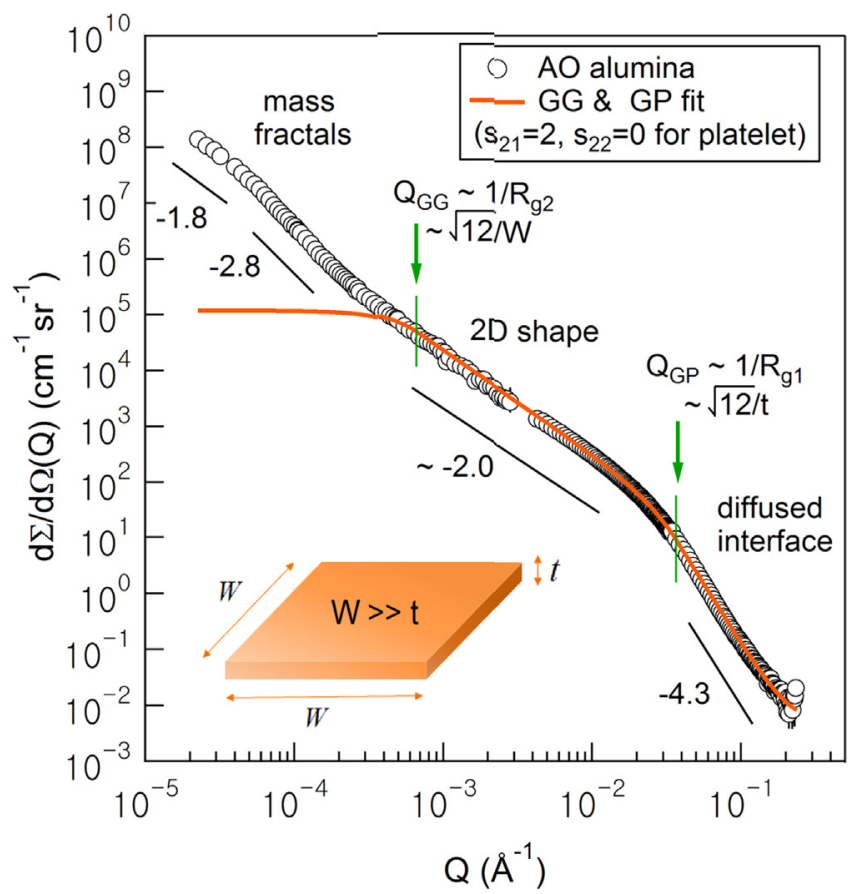

Fig. 2. USANS and SANS profiles (open circle) of pure AO alumina and generalized Guinier-Power and Guinier-Guinier fit (line) with $s_{21}=2, s_{22}=0$. Inset is a simplified platelet with the same lateral dimension. 
Table 2

Characterization of $\gamma-\mathrm{Al}_{2} \mathrm{O}_{3}$ particles with neutron scattering using GP and GG fittings ${ }^{\mathrm{a}}$.

\begin{tabular}{|c|c|c|c|c|c|c|c|c|c|c|}
\hline Sample & Shape & $s_{\mathrm{i} 1}$ & $s_{\mathrm{i} 2}$ & $p$ & $Q_{\mathrm{GP}}\left(\AA^{-1}\right)$ & $Q_{\mathrm{GG}}\left(\AA^{-1}\right)$ & $R_{\text {gi1 }}(\AA)$ & $R_{\text {gi2 }}(\AA)$ & \multicolumn{2}{|c|}{ Dimensions ( $\mathrm{A})$} \\
\hline AA & Rod & $\begin{array}{l}s_{11} \\
1\end{array}$ & $\begin{array}{l}s_{12} \\
0\end{array}$ & 4.2 & 0.10506 & $1.0384 \times 10^{-2}$ & $\begin{array}{l}R_{\mathrm{g} 11} \\
16.8\end{array}$ & $\begin{array}{l}R_{\mathrm{g} 12} \\
120\end{array}$ & $\begin{array}{l}D(\AA) \\
47.5\end{array}$ & $\begin{array}{l}L(\AA)(L \gg r) \\
411.6\end{array}$ \\
\hline $\mathrm{AO}$ & Platelet & $\begin{array}{l}s_{21} \\
2\end{array}$ & $\begin{array}{l}s_{22} \\
0\end{array}$ & 4.3 & 0.03723 & $6.6361 \times 10^{-4}$ & $\begin{array}{l}R_{\mathrm{g} 21} \\
31.8\end{array}$ & $\begin{array}{l}R_{\mathrm{g} 22} \\
2500\end{array}$ & $\begin{array}{l}t(\AA) \\
110^{\mathrm{b}}\end{array}$ & $\begin{array}{l}W(\AA)(W \gg r) \\
8659^{c}\end{array}$ \\
\hline
\end{tabular}

${ }^{a}$ Definitions of symbols are from Table 1. $D$ : diameter of rods, $t$ : thickness of platelets, $L$ : length of rods, $W$ : width of platelets.

b The thickness of a primary scatter can be from an average thickness of 2-3 layered platelets (see Table 3 and main text).

c The width of AO estimated by TEM was approximately $W=200-400 \AA$.

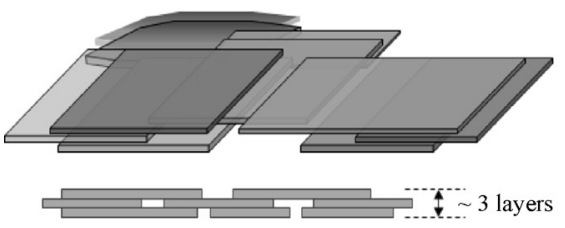

(a)

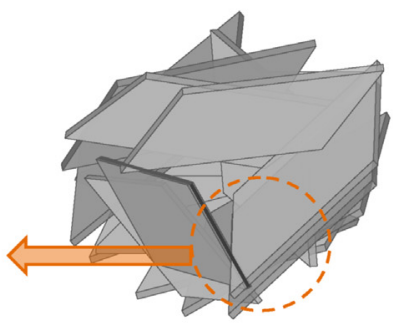

(b)

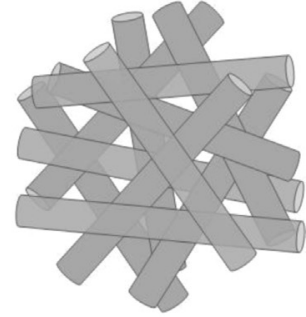

(c)

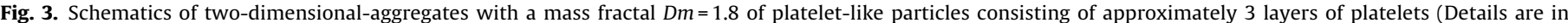

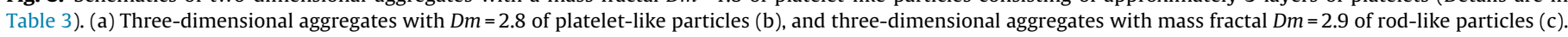

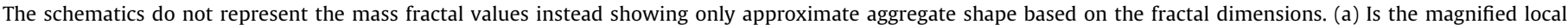
structure (dotted circle) of (b).

$2<p<3$ indicates a mass fractal arrangement in three-dimensional space while $1<p<2$ does a mass fractal in two-dimensions. $0<p<1$ represents a mass fractal in one-dimension without connection. The lowest mass fractal with connection is $p=1$, which is a line [17]. Fig. 3 shows the schematics of aggregations of the AA and AO alumina based on power laws.

The platelet-like AO alumina seem to cluster in a twodimensional manner, i.e., stacking a few layers (Fig. 3(a)) in a small scale followed by three-dmensional aggregates (Fig. 3(b)) in a larger scale. On the other hand, the rod-like AA alumina can aggregate in a three-dimensional manner (Fig. 3(c)) in broad size scales of approximately 3 orders from nanometer to micrometer or can have highly rough surfaces [18]. The power index $p=3$ is a boundary to distinguish mass fractals, $1<p<3$, and surface fractals, $3<p<4$. Since geometrically $p=3$ means a three-dimensional Euclidean shape, it is hard to believe that the aggregates of AA rod-like particles have a Euclidian shape. Therefore, $p=3$ is from the surface of particle aggregates (the size of aggregates are out of the USANS measurement), which can be roughly comparable to a mass fractal [18].

Negative deviation (i.e., the slope greater than 4.0, $p \sim 4.3$ ) from the Porod scatterings at the high $Q$ region, i.e. beyond $Q_{G 1 P}$, of both $\mathrm{AA}$ and $\mathrm{AO}$ alumina indicates that the boundary between the alumina crystallite particles and air is not sharp, but diffused [19-21]. Since both AA and AO alumina show the same slope, their diffusing interfaces are identical. Besides the same surface structure, XPS showed negligible difference in the chemical and electronic states of aluminum and oxygen atoms on the surface of the two types of alumina powders (see Supplementary information, Fig. S1.). Therefore, we can neglect surface effect to investigate the shape dependence on the catalytic activity of since the surfaces of AA and AO alumina are identical in both physical structure and chemical nature.

\subsection{Microscopic observation and aggregate behavior of alumina particles}

Microscopic structures of the two types of the $\gamma$-alumina powder (pure-AA and pure-AO) were observed with TEM and compared with characterizations based on the USANS and SANS measurements in the previous section. The TEM images (Fig. 4) show expected morphologies from the USANS and SANS analysis including the large aggregates, which also confirms that the scattering entities of USANS-SANS measurements are from the particles instead of the irregular shaped pores between $\gamma$-alumina crystallite particles.

Fig. 4(a) shows microscopic structures of pure AO. As was expected from the USANS and SANS measurements (Fig. 2 and Table 2), platelet-like shapes were observed. All of the observed platelets were lying flat perpendicular to the TEM e-beam, frequently piled onto each other as shown in darker area in TEM. The typical size of the platelets was 200-400 $\AA$ wide. The estimated width $(W)$ of the platelets by the USANS-SANS measurements was 8000-9000 $\AA$ (Table 2), which is probably from the overall size of two-dimensional aggregates of individual platelets as shown in TEM rather than the single platelet. The USANS scattering of pure AO in previous section showed also two-dimensional aggregates (Fig. 3(a)) in larger size. Fig. 4(b) shows microscopic structures of pure AA. Again, rod-like shapes were observed with 30-50 A cross sectional diameter and 300-500 $\AA$ in length, as was expected from the USANS and SANS measurements (Fig. 1 and Table 2). The shape and size are correlated reasonably well with the USANS-SANS analysis in Table 2.

Specific surface areas $(S / V)$ of $\gamma$-alumina can be estimated from the parameters obtained by USANS and SANS measurements as follows. AA with rod-like shapes: $(S / V)_{\text {rod }}=2(L+r) / r L$, AO with platelet-like shapes: $(S / V)_{\text {platelet }}=4(a+b+h) / a b h \approx 2(L+r) / r L$. $(S / V)$ was converted to the unit of $\mathrm{m}^{2} / \mathrm{g}=(1 / \mathrm{cm}) / \mathrm{g} / \mathrm{cm}^{3} \times(1 / 100)^{2}$ and the mass density of $\rho=3.65\left(\mathrm{~g} / \mathrm{cm}^{3}\right)$ of alumina was used [22]. Those estimated values were compared with BET surface area values and estimations from TEM images (Table 3). The number of platelet layers of AO alumina can be estimated by comparing specific surface area $(S / V)$ from the USANS-SANS and BET measurements in Table 3. Unlike pure AA, the specific surface areas of pure AO determined from the USANS-SANS $\left(51 \mathrm{~m}^{2} / \mathrm{g}\right)$ and BET $\left(130 \mathrm{~m}^{2} / \mathrm{g}\right)$ showed a large discrepancy (Table 3$)$, which can be attributed to the thickness of overlapped platelets. The TEM image 

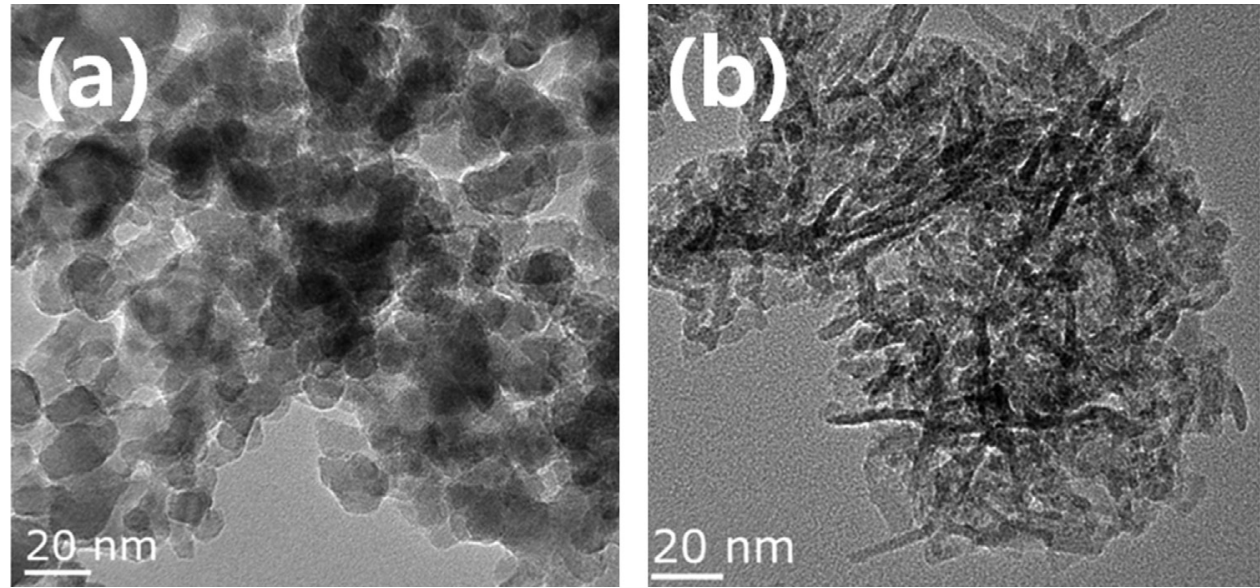

Fig. 4. (a) TEM image of pure-AO, (b) pure-AA.

Table 3

Specific surface areas $\left(S / V\right.$, unit: $\left.\mathrm{m}^{2} / \mathrm{g}\right)$ from different methods.

\begin{tabular}{llll}
\hline Sample & USANS-SANS & TEM & BET \\
\hline AA & 244 & $164-237$ & 255 \\
AO & $51^{\mathrm{a}}(101-150)^{\mathrm{a}}$ & $-^{\mathrm{b}}$ & 130 \\
\hline
\end{tabular}

a The $51 \mathrm{~m}^{2} / \mathrm{g}$ was obtained assuming the thickness of 2-3 layered platelets. Corresponding specific surface area of a single layer is $101-150 \mathrm{~m}^{2} / \mathrm{g}$. Mass density of $3.65\left(\mathrm{~g} / \mathrm{cm}^{3}\right)$ of alumina was used [22].

b The $S / V$ of AO from TEM could not be determined because of the lack of thickness information.

of AO shows stacks of multiple layers (darker color in Fig. 4(a)). The USANS-SANS could have measured the multiple layers of the platelet as a primary scatter at $Q_{\mathrm{GP}}$. When the platelet-like alumina consist of 2-3 layers, the thickness of $110 \AA$ of a multilayer is equivalent to a $55-35 \AA$ thickness of a single platelet layer and corresponds to a specific surface area of $S / V=101-150 \mathrm{~m}^{2} / \mathrm{g}$ which agrees with the ratio $S / V=130 \mathrm{~m}^{2} / \mathrm{g}$ obtained by the BET method. The possibility of spherical shapes for the AO alumina was investigated because the TEM images are two dimensional projections of three dimensional objects. However, spherical shapes were excluded based on the following analysis. The USANS and SANS measurements of bulk AO powder samples did not show the Guinier region of spherical shape with Eqs. (1) and (4). Instead, the scattering profiles as shown in Fig. 2 show a typical feature of platelet-like shape, slope $\sim-2$, appearing in Eqs. (2) and (4) over two decades of scattering vectors as well as two Guinier regions related to thickness and width of platelet-like shape. Detailed quantitative characterizations and modeling of the USANS and SANS were described in the previous section.

A question is raised on why AA and AO particles have different aggregation morphologies. This can be explained with difference in the van der Waals forces between the individual alumina particles.

The van der Waals force, $F_{\text {planar }}$, between planar surfaces can be written as [23]

$\frac{F_{\text {planar }}}{\text { area }}=-\frac{H}{6 \pi d_{\mathrm{pl}}^{3}}$,

where $H$ is the Hamaker constant, and $d_{\mathrm{pl}}$ is the distance between the surfaces. The van der Waals force, $F_{\text {rod }}$, between two identical and parallel cylindrical rods can be written as [23]

$F_{\text {rod }}=-\frac{H}{16} \frac{L \sqrt{R}}{d_{\text {rod }}^{5 / 2}}$,

where $H$ is the Hamaker constant, $d_{\text {rod }}$ is the distance between the rods, $L$ is the length of the rods, and $R$ is the radius of the rods. $H$ is the same for both cases (alumina-air-alumina). From the USANS/SANS analysis and TEM observation, we estimate $L \sim 400 \AA, R \sim 20 \AA$, and the area of a platelet AO particle $\sim 90,000 \AA^{2}$. With these numbers, we can compare the strength of the two forces at the same distance $d$ (i.e., $d_{\mathrm{pl}}=d_{\text {rod }}$ );

$\frac{F_{\text {planar }}}{F_{\text {rod }}}=\frac{8}{3 \pi} \frac{\text { area }}{L \sqrt{R} \sqrt{d}} \approx \frac{43}{\sqrt{d}}$.

Therefore, the two forces have similar strength when the interparticle distance $d$ is $1849 \AA$. When $d<1849 \AA$, $F_{\text {planar }}$ is stronger than $F_{\text {rod. }}$. For example, when $d \sim 10 \AA, F_{\text {planar }}$ is more than 10 times stronger than $F_{\text {rod }}$. This comparison suggests that the way rod-like AA particles aggregate is very different from that of platelet-like $\mathrm{AO}$ particles. Platelet-like AO particles aggregate platelet over platelet with strong van der Waals forces between the surfaces, forming stacks consisting of 2-3 individual platelets (Figs. 3 and 4(a)). The outer surface of such aggregate will consist of terraces with a small number of steps and kinks. On the other hand, the individual rodlike AA particles randomly aggregate (Figs. 3 and 4(b)) due to much weaker van der Waals forces.

\subsection{Deposition characteristics of Pt nanoparticles on alumina supports}

To find a correlation between the support shape and deposition characteristics of Pt catalyst nanoparticles, we deposited Pt on AA and AO by APD and checked the deposition behavior. The APD condition was $V=200 \mathrm{~V}, C=1800 \mu \mathrm{F}$, and $n=8815$ and it was applied to have about $2 \mathrm{wt} \%$ Pt loading on $4 \mathrm{~g} \gamma$-alumina powder. Actual Pt loading amounts measured by ICP-MS were $2.01 \mathrm{wt} \%$ for $\mathrm{AO}$ and 2.31 wt\% for AA. Fig. 5 shows TEM images of Pt deposited AO and AA. Fig. 5(a) shows the Pt/AO system. Small dark spots on AO platelets are Pt nanoparticles. Their average particle size was $17 \pm 7 \AA$. The deviation in particle size included measurement error mentioned in Section 2.3. Fig. 5(b) shows the Pt/AA system. Most of the Pt nanoparticles were smaller and the size distribution was narrower than those on AO. Their average particle size was $13 \pm 4 \AA$. More detailed size distribution of deposited Pt nanoparticles is shown in histograms in Fig. 6.

For Pt/AA, particle size in the range of $8-10 \AA$ was most common (Fig. 6(b)). The asymmetric size distribution with a tail in the direction of increasing size resembled the kinetic energy distribution of generated plasma [24]. This size distribution suggests that majority of deposited Pt nanoparticles did not merge with neighboring Pt nanoparticles and kept their intrinsic size with some depositing particles with high enough kinetic energy in the tail of high-end kinetic energy distribution. Those particles with high enough kinetic energy could have excessive energy after landing 

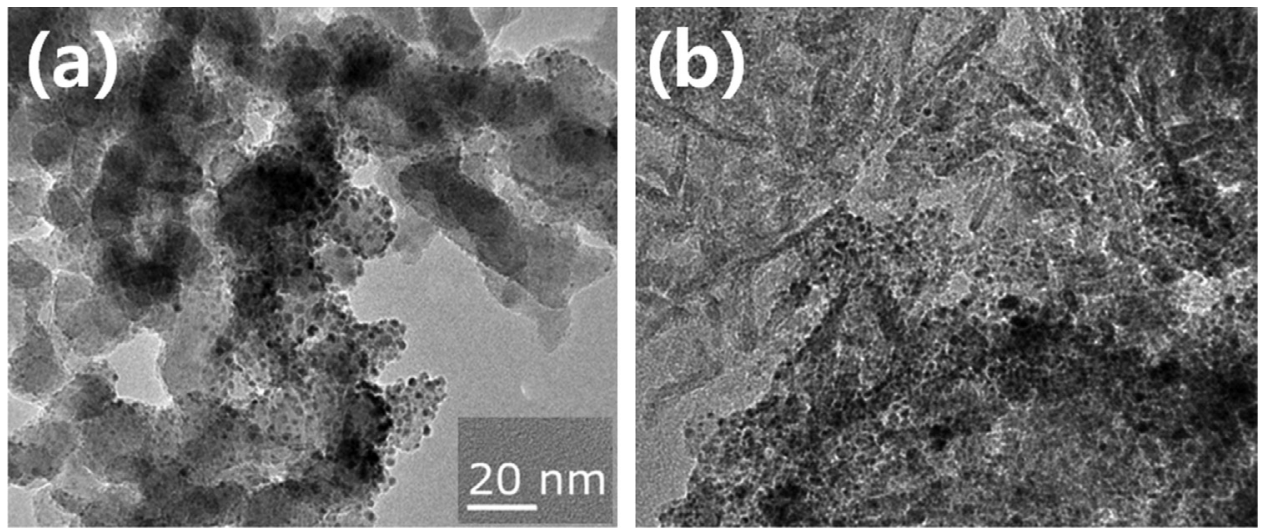

Fig. 5. TEM images of Pt deposited (a) AO and (b) AA. Scale bar in (a) applies for (b), too.
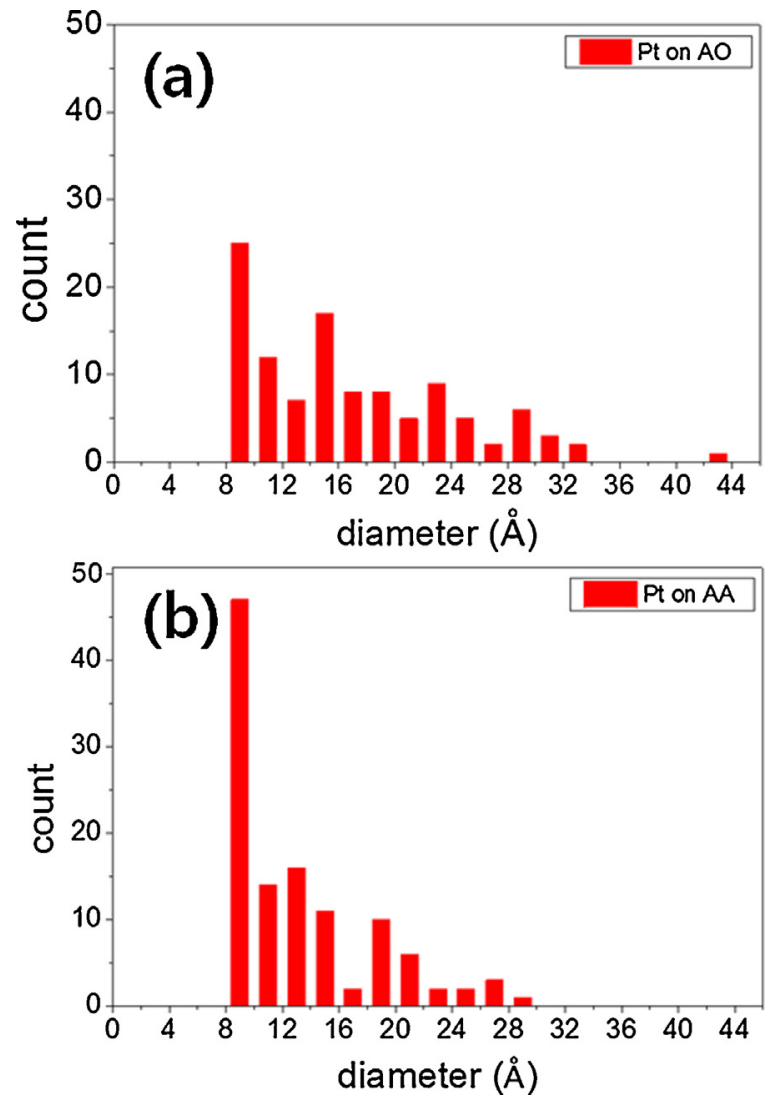

Fig. 6. Particle size distribution of deposited Pt nanoparticles in Fig. 5. (a) Pt on AO, (b) Pt on AA.

and diffuse around, meet other deposited particles, and merge, increasing the size of the finally stabilized particles. Thus, the number of particles that can actually diffuse around is determined by the activation energy barrier for the diffusion, resulting in the different degree of size distribution between AO and AA

The Pt particles on the AO support show broader size distribution, keeping its overall asymmetric distribution, a most frequent population at 8-10 $\AA$ and tailed up to approximately $35 \AA$. On the other hand, the Pt particles on the AA support show a narrow asymmetric distribution with much higher portion of the particles at 8-10 ̊ than that of Pt on AO and tailed only up to $25 \AA$. We believe that the difference of Pt size and its distribution between $A O$ and AA mainly comes from the difference in the shape of individual alumina particles and how they form larger clusters. The rod-like alumina (AA) have large dimensions along the length and much smaller dimension in the diameter while the platelet-like alumina (AO) have large dimensions in both laterals (see quantitative characterizations of USANS and SANS in Table 2). For individual alumina particles, Pt nanoparticles deposited on AO will see planar surfaces and their diffusion on the surfaces upon deposition will be facilitated by the low diffusion barriers of flat alumina surfaces. With repeated Pt deposition, they will have a higher chance of meeting already deposited Pt nanoparticles and forming bigger particles. On the other hand, Pt nanoparticles deposited on AA see higher diffusion barriers due to surface curvature and as a result, their diffusion upon deposition would be suppressed. The support curvature effect on the diffusion barrier of atomic particles is repeatedly reported in literature $[25,26]$. We cross-checked if the shape of support alumina changed during Pt deposition using AO alumina. USANS and SANS profiles confirm that the shape of AO did not change before and after catalyst loading (not shown).

\subsection{Catalytic reactivity comparison}

In the previous section, we investigated structural aspects of $\gamma$-alumina support and Pt nanoparticles deposited on them. In this section, we investigate catalytic activity of Pt/alumina systems described in the previous section with two different $\gamma$-alumina powders, rod-like and platelet-like. As explained in Section 2, the catalyst samples underwent a model reaction closely related to the three way catalyst (TWC) reactions ( $\mathrm{CO}, \mathrm{NO}, \mathrm{C}_{3} \mathrm{H}_{6}$ conversions) in a lean condition in a flow reactor [10]. Fig. 7 shows $\mathrm{CO}, \mathrm{NO}_{x}$, and $\mathrm{C}_{3} \mathrm{H}_{6}$ conversion rates of the two samples as a function of temperature.

In general, in a TWC reactor with the reactant gases in a lean mixture, it is known that oxidation reactions such as $\mathrm{CO}$ and $\mathrm{C}_{3} \mathrm{H}_{6}$ oxidation reach $100 \%$ conversion rate with high enough temperature due to abundant adsorbed oxygen atoms on the catalyst surface, while reduction reaction of $\mathrm{NO}_{x}$ to $\mathrm{N}_{2}$ is not so efficient due to the lack of reducing agents on the catalyst surface [11]. When NO molecules adsorb on the catalyst surface sites, they dissociate and remain as $\mathrm{N}$ atoms and $\mathrm{O}$ atoms on the surface. If there are enough adsorbed $\mathrm{CO}$ molecules available on the surface, they will react with the dissociated $\mathrm{O}$ atoms, forming the $\mathrm{CO}_{2}$ molecules and desorbing the surface. These reduction reactions let surface sites free for subsequent adsorption of NO molecules. This cycle enables NO conversion in a lean condition [12]. Therefore, NO conversion rate increases with increasing $\mathrm{CO}$ conversion rate to some extent, then decreases sharply with the CO conversion rate reaching $100 \%$, when little $\mathrm{CO}$ molecules are available on the catalyst surface. As shown in Fig. 7, this general tendency was observed in our reactions, too. However, catalytic efficiencies were different depending 

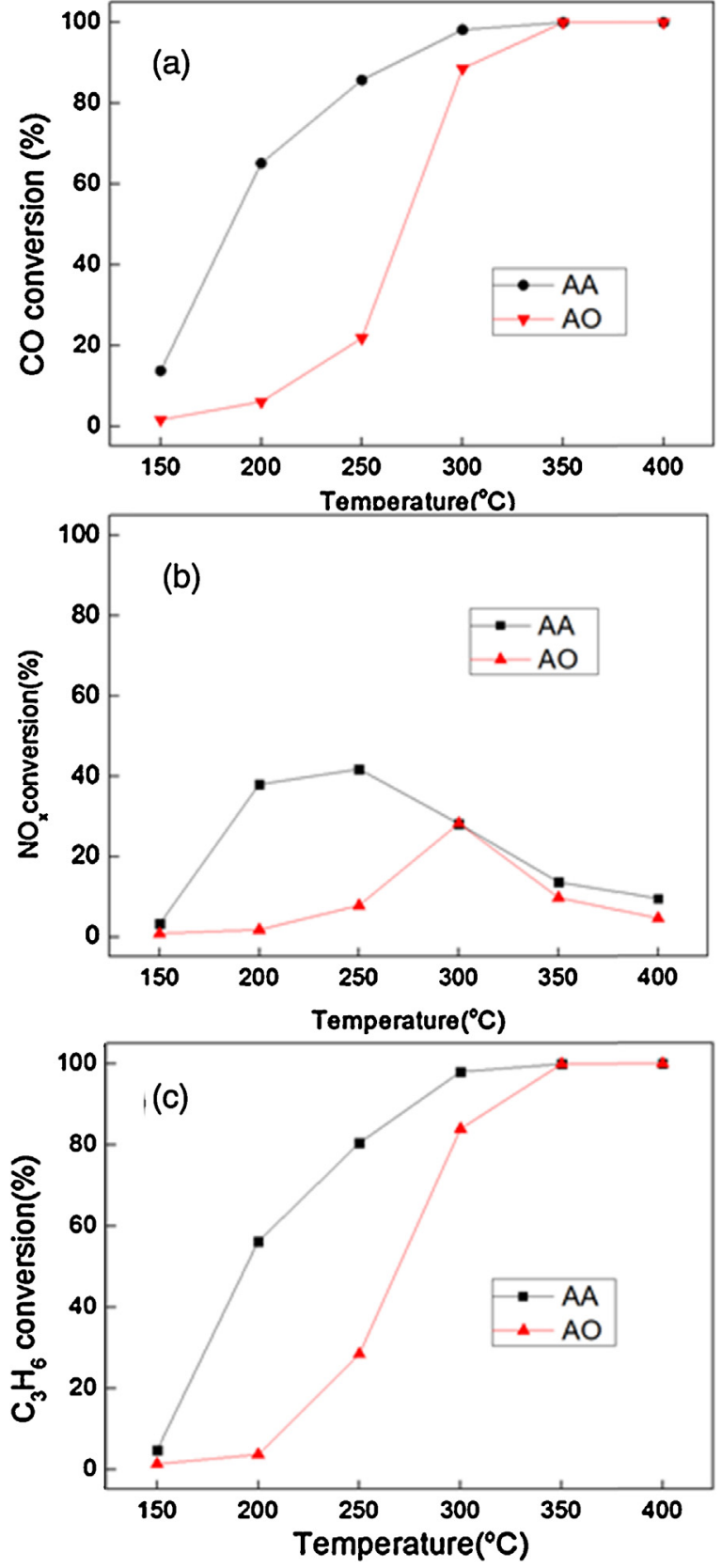

Fig. 7. Conversion rate comparisons for TWC model reactions. (a) CO oxidation, (b) NO reduction, (c) $\mathrm{C}_{3} \mathrm{H}_{6}$ oxidation.

on the shape of the alumina support and the degree of dispersion of deposited Pt nanoparticles.

Overall, AA samples showed higher conversion rates than those of AO samples. This observation is in line with the observation in Figs. 5 and 6 that the dispersion of Pt nanoparticles on AA was better than that on AO, leading to higher active surface area on AA. Especially, low temperature efficiency of the AA sample was particularly higher than those of the AO samples. In Fig. 7(a), catalytic efficiency of Pt-AA for CO oxidation was about $62 \%$, while that of Pt-AO was about $23 \%$ at $250^{\circ} \mathrm{C}$. In other words, Pt-AA had more than $100 \%$ higher catalytic efficiency than Pt-AO at $250^{\circ} \mathrm{C}$. This superior catalytic efficiency was observed at $200-250^{\circ} \mathrm{C}$. Other reactions such as $\mathrm{NO}$ reduction (Fig. 7(b)) and $\mathrm{C}_{3} \mathrm{H}_{6}$ oxidation (Fig. $7(\mathrm{c}$ )) showed similar tendencies. Given the same materials of the catalyst $(\mathrm{Pt})$ and support ( $\gamma$-alumina), the exposed surface area of the deposited Pt nanoparticles will be one of the most important factors for catalytic activity. We estimated the surface/volume ratio of each deposition condition based on the Pt size distribution in Fig. 6. We assumed a spherical shape for each Pt nanoparticle and calculated surface area and volume of the spheres. Then, we summed up half of the surface area and volume of each nanoparticle to get the total surface area and volume. While the $S / V$ for the AO samples were 6.0 for Pt-AO, that for AA was 7.9 (unit: $3 \times 10^{-7} / \mathrm{m}$ ). Indeed, the $S / V$ for $A A$ was higher than those for AO. This estimation indicates that the dispersion of Pt nanoparticles on the rod-like AA was better than that on the platelet-like AO, leading to higher surface area for a given amount of Pt deposition.

These results suggest that, even for the same catalytic support material, catalytic activity can be strongly dependent on the characteristic size and shape of the material and how they aggregate. This difference leads to the difference in the degree of dispersion of the deposited catalyst nanoparticles and eventually in the catalytic activity.

\section{Conclusions}

Two $\gamma$-alumina powders, AA alumina and AO alumina, showed different shapes, rod-like and platelet-like, which were characterized in 4 orders of length scales, from nanometers to micrometers, using USANS and SANS. The aggregation of the alumina particles were also affected by the shape of individual alumina particles. Platelet-like alumina consisted of stacks of 2-3 layers of individual platelets and aggregates of the stacks. Rod-like alumina formed random aggregation. Loading behavior and catalytic activity of catalyst Pt nanoparticles on the $\gamma$-alumina were affected by the alumina shapes and how they aggregated. Rod-like shapes with smaller overall characteristic sizes showed higher catalytic activity than those with platelet-like shapes with bigger characteristic sizes. This superior catalytic performance of the smaller characteristic size and shape is thought to be due to better dispersion of catalyst nanoparticles on them. The shape, the way how they aggregate, and specific surface area of catalytic support materials are important parameters for determining catalytic activity as much as chemical properties of the support materials.

\section{Acknowledgments}

This research was supported by several grants from Korea Institute of Science and Technology (KIST) (2E25371, 2V03990, 2V04081). MHK also acknowledges partial financial support from KAERI-KIST Collaborative Project (2N40270). We thank Dr. Baek Seok Seong and Dr. Eunjoo Shin of the HANARO at Korea Atomic Energy Research Institute (KAERI) for experiment support of $18 \mathrm{~m}$ SANS. We would like to thank Boualem Hammouda at the NIST Center for Neutron Research (NCNR) for reviewing this manuscript.

\section{Appendix A. Supplementary data}

Supplementary data associated with this article can be found, in the online version, at http://dx.doi.org/10.1016/j.cattod.2015.05. 031

\section{References}

[1] I. Meusel, J. Hoffmann, J. Hartmann, J. Libuda, H.J. Freund, Size dependent reaction kinetics on supported model catalysts: a molecular beam/IRAS study of the CO oxidation on alumina-supported Pd particles, J. Phys. Chem., B 105 (2001) 3567-3576.

[2] E. Roduner, Size matters: why nanomaterials are different, Chem. Soc. Rev. 35 (2006) 583-592

[3] R.M. Rioux, H. Song, J.D. Hoefelmeyer, P. Yang, G.A. Somorjai, High-surface-area catalyst design: synthesis, characterization, and reaction studies of platinum nanoparticles in mesoporous SBA-15 silica, J. Phys. Chem., B 109 (2004) 2192-2202. 
[4] I. Balint, A. Miyazaki, K.-I. Aika, Effect of platinum morphology on lean reduction of NO with $\mathrm{C}_{3} \mathrm{H}_{6}$, Phys. Chem. Chem. Phys. 6 (2004) 2000-2002.

[5] J. Park, Current Trends of Surface Science and Catalysis, Springer, New York, NY, 2014

[6] M. Trueba, S.P. Trasatti, $\gamma$-Alumina as a support for catalysts: a review of fundamental aspects, Eur. J. Inorg. Chem. 2005 (2005) 3393-3403.

[7] M. Digne, R. Revel, M. Boualleg, D. Chiche, B. Rebours, M. Moreaud, B. Celse, C. Chanéac, J.P. Jolivet, Innovative characterizations and morphology control of $\gamma$-AlOOH boehmite nanoparticles: towards advanced tuning of $\gamma-\mathrm{Al}_{2} \mathrm{O}_{3}$ catalyst properties, in: E.M. Gaigneaux, M. Devillers, S. Hermans, P.A. Jacobs, J.A. Martens, P. Ruiz (Eds.), Studies in Surface Science and Catalysis, Elsevier, Amsterdam, 2010, pp. 127-134

[8] L.D. Hart, E. Lense, Alumina Chemicals: Science and Technology Handbook, American Ceramic Society, Westerville, OH, 1990.

[9] S.H. Kim, C.-H. Jung, N. Sahu, D. Park, J.Y. Yun, H. Ha, J.Y. Park, Catalytic activity of $\mathrm{Au} / \mathrm{TiO}_{2}$ and $\mathrm{Pt} / \mathrm{TiO}_{2}$ nanocatalysts prepared with arc plasma deposition under CO oxidation, Appl. Catal., A: Gen. 454 (2013) 53-58.

[10] S.i. Matsumoto, Recent advances in automobile exhaust catalysts, Catal. Today 90 (2004) 183-190.

[11] N. Takahashi, H. Shinjoh, T. Iijima, T. Suzuki, K. Yamazaki, K. Yokota, H. Suzuki, N. Miyoshi, S.-I. Matsumoto, T. Tanizawa, T. Tanaka, S.-S. Tateishi, K. Kasahara, The new concept 3-way catalyst for automotive lean-burn engine: $\mathrm{NO}_{x}$ storage and reduction catalyst, Catal. Today 27 (1996) 63-69.

[12] D. Chatterjee, O. Deutschmann, J. Warnatz, Detailed surface reaction mechanism in a three-way catalyst, Faraday Discuss. 119 (2002) 371-384.

[13] O. Glatter, O. Kratky, Small Angle X-ray Scattering, Academic Press, London; New York, 1982.

[14] B. Hammouda, A new Guinier-Porod model, J. Appl. Crystallogr. 43 (2010) 716-719.

[15] P. Mittelbach, Zur roentgenkleinwinkelstreuung verdunter kolloider systeme. VIII. Diskussion des streuverhaltens regelmaessiger koerper und methoden zur bestimmung von groesse und form kolloider teilchen, Acta Phys. Aust. 19 (1964) 53-102.

[16] S.R. Kline, Reduction and analysis of SANS and USANS data using IGOR Pro, J. Appl. Crystallogr. 39 (2006) 895-900.

[17] J.E. Martin, A.J. Hurd, Scattering from fractals, J. Appl. Crystallogr. 20 (1987) $61-78$.

[18] K.D. Keefer, D.W. Schaefer, Growth of fractally rough colloids, Phys. Rev. Lett. 56 (1986) 2376-2379.

[19] C. Vonk, Investigation of non-ideal two-phase polymer structures by small-angle X-ray scattering, J. Appl. Crystallogr. 6 (1973) 81-86.

[20] W. Ruland, Small-angle scattering of two-phase systems: determination and significance of systematic deviations from Porod's law, J. Appl. Crystallogr. 4 (1971) 70-73.

[21] M.-H. Kim, Modified Porod's law estimate of the transition-layer thickness between two phases: test of triangular smoothing function, J. Appl. Crystallogr. 37 (2004) 643-651 (Official contribution of the National Institute of Standards and Technology; not subject to copyright in the United States).

[22] http://www.reade.com/products/35-oxide-metallic-powders//.

[23] J.N. Israelachvili, Intermolecular and Surface Forces, third ed., Academic Press Burlington, MA, 2011.

[24] W.D. Davis, H.C. Miller, Analysis of the electrode products emitted by dc arcs in a vacuum ambient, J. Appl. Phys. 40 (1969) 2212-2221.

[25] A.V. Krasheninnikov, K. Nordlund, P.O. Lehtinen, A.S. Foster, A. Ayuela, R.M. Nieminen, Adsorption and migration of carbon adatoms on carbon nanotubes: density-functional $a b$ initio and tight-binding studies, Phys. Rev. B: Condens. Matter 69 (2004) 073402.

[26] M.-C. Wu, C.-L. Li, C.-K. Hu, Y.-C. Chang, Y.-H. Liaw, L.-W. Huang, C.-S. Chang, T.-T. Tsong, T. Hsu, Curvature effect on the surface diffusion of silver adatoms on carbon nanotubes: deposition experiments and numerical simulations, Phys. Rev. B: Condens. Matter 74 (2006) 125424. 